\title{
Prevalence of HIV in pregnant women in a rural area of Maharashtra, India Tamboli $S S^{1}$, Raju $R^{2}$, Nagoba $B S^{3}$
}

Dear Editor,

The PPTCT program aims to prevent peri-natal transmission of HIV from an HIV infected pregnant mother to her newborn baby. The program entails counseling and testing of pregnant women in the ICTCs. Pregnant women found to be HIV- positive are given a single dose of Nevirapine tablet at the time of labor; their newborn babies also get a single dose of Nevirapine syrup within 72 hours after births to prevent transmission of HIV from mother to child ${ }^{1}$. According to National AIDS Control Organization (NACO), it is estimated that about 30,000 infants acquire HIV infection every year ${ }^{2}$. National AIDS Control Organization of India initiated phase 1 feasibility study on the prevention of mother to child transmission using the short course regimen of Azidothymidine (AZT) and phase 2 of this project using Nevirapine (NVP) had been initiated in the year 1999 at 11 centers ${ }^{1,3}$.

The six year data of PPTCT program in a rural area of India from Jan 2005 to Dec 2010 was analyzed. Of the total 9435 antenatal care (ANC) patients counseled for PPTCT, 8153 (86.41\%) women were tested for HIV following NACO guidelines. ${ }^{1}$ Out of these $34(0.41 \%)$ women were found to be HIV positive. Out of 34 women, 19 (55.88\%) delivered in the hospital. All women delivered in the hospital received Nevirapine tablet. It was found that no transmission of HIV was seen in all children, as confirmed at the age of 18 months by using NACO guidelines. Thus, this strategy prevented transmission of HIV from mother to child. In our study all out of 8153 agreed to undergo HIV testing. 34 were tested positive, giving prevalence of $0.41 \%$. This is less as compared to other reports from rural areas from different states of India ${ }^{4,5}$.

HIV infection is increasing steadily in majority of the countries in the world. India is not an exception to this phenomenon. HIV infection is no longer restricted to the core groups practicing unsafe behaviors in six Indian states - Maharashtra, Tamil Nadu, Karnataka, Manipur Andhra Pradesh and Nagaland but is also establishing itself in general population. Utilization of the PPTCT services can be improved by creating awareness among the pregnant women regarding the need and benefits of getting tested. Efforts to increase the level of HIV testing in ANC, acceptance of PPTCT services, disclosure to partners and couple testing remain a priority. Increased counseling, treatment and support of women identified as HIV infected should improve acceptance of PPTCT services and subsequently reduce pediatric HIV. The need of the hour is to provide universal access to these services by involving NGO's and the private sector.

Keywords: PPTCT, Pregnant women, HIV.

Conflict of interest- None to declare

\section{References:}

1. NACO: Revised PPTCT Training Curriculum Trainer Manual (India), New Delhi, India; 2004

2. National AIDS Control Organization. NACP-III. Available from: http://www.ijmm.org/www

3. Kunte A, Misra V, Paranjape R, etal. HIV Seroprevalence and awareness about AIDS among pregnant women in rural areas of pune district, Maharashtra, India. Indian $J$ Med Res 1999; 110:115-22
4. M Dash, S padhi, P. Panda etal. PPTCT of HIV : Two and a half year experience at MKCG medical college, Berhampur, India, Indian J Med Microbiol 2009; 27: 276277

5. Mandal S, Bhattacharya RN, Chakraborty Metal. Evaluation of the prevention of Parent to Child Transmission Program in a Rural tertiary Care Hospital of West Bengal, India. Indian J Community Med 2010; 35:491-494.

1. Sabiha S. Tamboli, Assistant Professor, Dept. of Microbiology.

2.Reena Raju, Associate professor, Dept. of Gynecology.

3. B.S Nagoba, Assistant Dean.

MIMSR Medical College, Latur, Maharashtra, India.

Corresponds to: Dr. Sabiha S. Tamboli, Assistant Professor,Dept. of Microbiology, MIMSR Medical College, Latur, Maharashtra, India. Email: microbiopharma@yahoo.com 